\title{
Competitive Ability and Fitness of Alternaria alternata Isolates Resistant to Qol Fungicides
}

\author{
G. S. Karaoglanidis, Y. Luo, and T. J. Michailides, Department of Plant Pathology, University of California, Davis, and Kearney Agri- \\ cultural Center, Parlier, CA 93648
}

\begin{abstract}
Karaoglanidis, G. S., Luo, Y., and Michailides, T. J. 2011. Competitive ability and fitness of Alternaria alternata isolates resistant to QoI fungicides. Plant Dis. 95:178-182.

Fungicides that act as quinone outside inhibitors (QoIs) constitute a fungicide group extensively used against Alternaria late blight of pistachio caused by Alternaria spp. However, developement of resistance to this fungicide class constitutes an important threat for the succesful control of the disease. This study was conducted to determine whether development of resistance to QoIs is associated with a fitness cost, by measuring several biological and epidemiological parameters and estimating the competitive ability in four QoI-resistant and four QoI-sensitive Alternaria alternata isolates. Fitness parameters measured were mycelial growth and spore production in vitro, disease latent period, aggressiveness, and spore production on detached pistachio leaves. The competitive ability of resistant isolates was assessed in coinoculation experiments with sensitive isolates on detached pistachio leaves, using

a real-time polymerase chain reaction assay technique. Fitness parameters between grouped QoI-resistant and QoI-sensitive isolates were not significantly different $(P=0.13,0.21,0.31$, and 0.27 for sporulation in vitro, mycelial growth, incubation period, and sporulation in vivo, respectively), while resistant isolates, as a group, showed a higher aggressiveness $(P=0.01)$ compared with the sensitive isolates. The data indicate that the resistant strains did not account for a fitness cost compared with the sensitive ones under the conditions of testing. The outcome of the competition experiments was isolate dependent. In two pairs, the resistance frequencies increased whereas, in the remaining two pairs of isolates, resistance frequency decreased, suggesting that the resistant isolates were competitive similarly to the sensitive isolates.
\end{abstract}

Alternaria late blight of pistachio (Pistacia vera L.), caused by Alternaria alternata (Fr.) Keissl., A. tenuissima (Kunze) Wiltshire, and $A$. arborescens E.G. Simmons, is one of the most important fungal diseases of pistachio in California. The disease is characterized by the development of large necrotic lesions on the foliage that progressively coalesce, leading to premature defoliation. On the fruit, the disease is characterized by the development of small necrotic spots on the hull of immature nuts, causing staining of the nutshells that lead to a reduction of the fruit quality (30).

Control of the disease is achieved by a combination of cultural practices (such as irrigation management and appropriate pruning to increase air movement and reduce relative humidity within tree canopy) and the use of fungicide spray applications. Fungicides registered for use against Alternaria late blight of pistachio in California include sterol demethylation inhibitors, quinone outside inhibitors (QoIs), and succinate dehydrogenase inhibitors (SDHIs) (2).

QoIs constitute a fungicide class that has been developed from natural fungicidal derivatives such as strobilurin A, oudemansin A, and myxothiazol A (5). The mechanism of action that this fungicide class possesses consists of inhibiting mitochondrial respiration by binding at the Qo site of cytochrome $b$. Inhibition of mitochondrial respiration is achieved by blocking the electron transport between cytochrome $b$ and cytochrome $c_{1}$, which leads to a disruption of the energy cycle (5). Azoxystrobin was the first QoI fungicide registered for use against Alternaria late blight disease in California pistachio early in 2000. Later, two additional QoIs,

Corresponding author: T. J. Michailides, E-mail: themis@uckac.edu

Current address of G. S. Karaoglanidis: Aristotelian University of Thessaloniki, Faculty of Agriculture, Plant Pathology Laboratory, P.O. Box 269, 54006, Thessaloniki, Greece.

Accepted for publication 1 October 2010.

doi:10.1094/PDIS-07-10-0510

(C) 2011 The American Phytopathological Society trifloxystrobin and pyraclostrobin, were included in the spray programs. After 2 to 3 years of azoxystrobin use with initially high efficacy against the pathogens, the first failures in controlling the disease were observed (23). More recently, dual resistance in $A$. alternata isolates to QoI and SDHI fungicides was reported $(2,3)$.

Resistance of Alternaria spp. to azoxystrobin has been attributed to a mutation in the cytochrome $b$ (cyt $b$ ) gene causing an amino acid substitution of glycine with alanine at codon 143 (G143A), $(20,21)$. This type of mutation is the predominant mutation conferring resistance to QoIs and has been shown to result in a complete loss of disease control in several other pathogens $(6,10,11,34)$. In addition, one more mutation leading to replacement of phenylalanine to leucine at position 129 (F129L) has been associated to resistance to QoIs (13). Moreover, another amino acid change, from glycine to arginine at position 137 (G137R), has also been linked to QoI resistance (34). Isolates carrying F129L or G137R express moderate resistance to QoIs. In contrast, isolates with G143A express high resistance.

Mutations associated with fungicide resistance may display deleterious pleiotropic effects, known as fitness cost, that become apparent in the absence of fungicide selection pressure $(14,33)$. Fitness can be defined as the survival and reproductive success of an allele, individual, or group (29). Evolution of fungicide resistance in fungal populations is largely dependent on the fitness of the strains that affects the dynamics of competition between resistant and sensitive strains, and this has important implications for disease management $(26,28)$. The evolution of fungicide resistance would be lessened if resistant subpopulations had lower parasitic or saprophytic fitness. In contrast, absence of fitness cost in the resistant fraction of the population would lead to a stable resistance frequency in the absence of fungicide pressure or to rapid development and evolution of resistance in the presence of fungicide pressure. The experimental data provided in the literature on the persistence of fungicide resistance make generalizations difficult. Several previous studies have shown that absence or presence of fungicide resistance-associated fitness cost is dependent on the fungal species and the fungicide class $(7,15,22,31,32)$. Although the experimental data are limited, it seems that fungal pathogens 
carrying the F129L mutation suffer a relative fitness cost (27), whereas the fitness of the G143A mutants appears to depend upon the pathogen (8).

Detailed knowledge of the fitness of the QoI-resistant strains is required in order to optimize Alternaria late blight management programs in pistachio orchards of California. Therefore, the objectives of this study were to (i) measure several fitness components such as mycelial growth, spore production in vitro and in vivo, and aggressiveness in QoI-resistant and -sensitive isolates of the pathogen; and (ii) determine the competitive ability of the resistant isolates in the absence of QoI selection pressure.

\section{Materials and Methods}

Fungal isolates and sensitivity determination. Four azoxystrobin-resistant and four azoxystrobin-sensitive single-spore isolates of A. alternata were used in the experiments. The isolates were collected during a monitoring program carried out in 2007, to determine the fungicide sensitivity of A. alternata isolates obtained from pistachio orchards grown in California to QoIs and the SDHI boscalid (3). Identification of A. alternata was performed according to a previously established protocol based on morphological characters (30). The azoxystrobin effective concentration that reduces spore germination by $50 \%\left(\mathrm{EC}_{50}\right)$ values were determined based on the inhibition of spore germination according to a procedure described previously (23). The eight selected isolates were either singly resistant to QoIs or were sensitive to both QoIs and SDHIs. Until use, the isolates were maintained on potato dextrose agar (PDA), (Oxoid, Basingstoke, UK) slants in a refrigerator at $4^{\circ} \mathrm{C}$.

DNA extraction and G143A mutation detection. DNA was extracted from the eight isolates used in the study and grown on PDA for 5 days following the procedure described by Ma et al. (23). The allele-specific primer pairs ARF4 (5'-ATGAGA GATGTAAATAATGGGTGAT-3') and ARR4 (5'-AAGGTTAGT AATAACTGTTGCAG-3') were used to amplify a 246-bp polymerase chain reaction (PCR) product from the resistant isolates, while the allele-specific primers ARF4 and ARS4 (5'-AAGGTT AGTAATAACTGTTGCAC-3') were used to amplify a similar product from the QoI-sensitive isolates. The two reverse primers ARR4 and ARS4 differed at the 3 ' end position, based on the single point mutation of GGT in the sensitive isolates to GCT in the resistant isolates. PCR amplifications were carried out following previously published procedures and conditions (23).

Fitness components. The following fitness components were determined for azoxystrobin-resistant and -sensitive isolates of $A$. alternata: (i) mycelial growth on PDA, (ii) spore production in vitro, (iii) incubation period, (iv) aggressiveness, and (v) spore production in vivo. All of the experiments were repeated in triplicate.

Mycelial growth. To measure mycelial growth, precultures for each isolate were established on PDA medium by transferring mycelial plugs. After 10 days of growth, four 5-mm mycelial plugs per isolate were removed from the margins of actively grown precultures and transferred to the center of fresh PDA plates for radial growth measurements. The plates were incubated at $22^{\circ} \mathrm{C}$ in the dark for 6 days and then the colony diameter of each isolate was measured. For each isolate, four replicate plates were prepared with one colony per plate.

Spore production in vitro. To determine the spore production of the isolates, PDA plates were inoculated with a 5-mm mycelial plug and incubated for 10 days at $22^{\circ} \mathrm{C}$ with a 14-h photoperiod provided by cool white fluorescent light. Then, sporulating colonies were rinsed with $20 \mathrm{ml}$ of distilled water and the conidial suspension was filtered through double-layered cheesecloth. The spore concentration in the suspension was measured with the aid of a hemacytometer and expressed as number of conidia per square millimeter of the PDA culture. Four replicate droplets were counted for each plate and four plates were used per isolate.

Latent period and aggressiveness. The disease latent period and the aggressiveness of the isolates was determined by inoculating detached pistachio leaves according to the method of Pryor and Michailides (30). In total, 30 detached, fully expanded leaves of the cv. Kerman were inoculated per isolate. The leaves were obtained from mature trees grown in the experimental field of Kearney Agricultural Center in Parlier, CA. Before inoculation, the leaves were surface sterilized by immersing them in a $1 \%$ sodium hypochlorite solution for $1 \mathrm{~min}$. After removing them from the disinfectant solution, they were rinsed three times in steriledeionized water and subsequently air dried. The leaves were then wounded on the midrib at three points with a razor blade and placed on wire mesh platforms in plastic boxes ( 23 by 31 by $10 \mathrm{~cm}$ [length by width by height]). Inoculation was carried out on the wounded leaves by placing $20 \mu \mathrm{l}$ of a $10^{3} / \mathrm{ml} \mathrm{H}_{2} \mathrm{O}$ conidial suspension on each wound. Leaves used as control were inoculated with $20 \mu \mathrm{l}$ of sterile distilled water. Tap water $(20 \mathrm{ml})$ was added in each box and then covered to maintain high relative humidity. The containers with the inoculated leaves were incubated at $20^{\circ} \mathrm{C}$ for 10 days. During these 10 days, the inoculated leaves were examined daily in order to determine the time period between inoculation and appearance of the first lesions (latent period) on each inoculated leaf. Ten days after the inoculation, the infection was recorded by measuring the lesion diameter on each leaf. The scoring was based on a four-point rating system as follows: $1=$ no lesion, $2=$ lesions $<1 \mathrm{~mm}$ in diameter, $3=$ lesions 1 to $5 \mathrm{~mm}$ in diameter, and $4=$ lesions $>5 \mathrm{~mm}$ in diameter.

Spore production in vivo. After measurement of aggressiveness, leaf segments (about $20 \mathrm{~mm}^{2}$ ) with sporulating lesions were removed with the aid of a 5-mm-diameter cork borer. One segment was removed from each leaf and 10 leaves were tested per isolate. Each leaf segment was placed in $3 \mathrm{ml}$ of distilled water in plastic tubes, vortexed for $20 \mathrm{~s}$ to detach the conidia from the lesion, and the leaf segment was subsequently removed. Droplets of the conidial suspension were placed on the hemacytometer and the number of conidia was counted. Four replicate droplets were counted for each tube. The number was expressed as the number of conidia per square millimeter of diseased leaf surface.

Competition experiments. Experimental design. Conidia of all isolates were produced as described above. Mixed-isolate inocula were prepared by mixing appropriate volumes of conidial suspensions of R1 with S1, R2 with S2, R3 with S3, and R4 with S4 isolates, so as to produce suspensions containing 100\%R:0\%S, 80\%R:20\%S, 50\%R:50\%S, 20\%R:80\%S, and 0\%R:100\%S conidia. All suspensions contained a final concentration of $1 \times 10^{3}$ conidia/ml. Detached pistachio leaves were inoculated and incubated as described previously (30). For each pair of isolates and for each conidial ratio, 10 replicate leaves were inoculated at three points. In addition, a set of 10 leaves, serving as a control, were inoculated with $20 \mu \mathrm{l}$ of sterile distilled water. After 10 days of incubation, the sporulating lesions were removed from each leaf to prepare the inoculum for the next disease cycle. The removed lesions from each leaf were added into $10 \mathrm{ml}$ of sterilized tap water and gently shaken to dislodge the conidia. The resulting suspension was used to inoculate a new set of leaves. In this way, a new disease cycle was started. Before adding the removed lesions into the water to create a new conidial suspension, 20 leaf segments per leaf removed with a 5-mm cork borer were separated for DNA extraction. The experiment was terminated after four disease cycles and was repeated twice.

Quantification of the resistance allele using real-time PCR. The determination of the percentage of azoxystrobin-resistant conidia in the mixed populations was carried out after the end of the second, third, and fourth disease cycle using a real-time PCR assay. DNA was extracted from sporulating leaf segments using the FastDNA extraction kit (QbioGene, Carlsbad, CA), according to a procedure previously described $(18,21)$. The above-mentioned primer pairs ARF4 + ARR4 and ARF4 + ASR were used for amplifications. The real-time PCR amplifications were performed in the DNA Engine Opticon 2 System (MJ Research, Waltham, MA) using the SYBR Green I fluorescent dye detection. Reagent quantities, amplification conditions, and standard curves were as those 
described by Luo et al. (18). For each sample, two real-time PCR amplifications were conducted in the same run: one for the determination of the resistant 143A allele, using the primer pair ARF4 + ARR4, and a second for the determination of the sensitive G143 allele using the primer pair ARF4 + ASR. The frequency of the G143A allele in each sample (FA) was calculated using the formula $F A=Q_{A} /\left(Q_{A}+Q_{G}\right)$, where $Q_{A}$ is the quantity (picograms) of template DNA with the resistant allele determined with the primer pair ARF4 + ARR4, and $Q_{G}$ is the quantity of the template DNA with the sensitive allele determined with the primer pair ARF4 + ASR (18).

Data analysis. The $\mathrm{EC}_{50}$ values to azoxystrobin of each isolate were calculated by regressing the relative inhibition of conidial germination against the $\log _{10}$ fungicide concentrations. Data of the three independent replications for each component tested were pooled after testing homogeneity of variance using the Levene test and subjected in an analysis of variance, except for the aggressiveness data, for which the nonparametric analysis with Kruskal-Wallis test was conducted by using the NPAR1WAY procedure of SAS to determine the significance in aggressiveness between resistant and sensitive groups of isolates used in this study. Mean values of each isolate within sensitivity groups were compared using Fisher's protected least significant difference test while means among sensitivity groups were compared by the pairwise Student's $t$ test. In the competition experiments, the observed frequency (percent) of resistant isolates was compared with the initial frequency of resistant isolates by the pairwise Student's $t$ test. All the statistical analysis tests were performed using SAS (JMP; SAS Institute, Cary, NC).

\section{Results}

Azoxystrobin sensitivity and G143A mutation detection. Measurements of sensitivity to azoxystrobin showed that the four QoI-resistant isolates had $\mathrm{EC}_{50}$ values higher than $200 \mu \mathrm{g} \mathrm{ml}^{-1}$, while sensitivity of the four azoxystrobin-sensitive isolates ranged from 0.15 to $0.22 \mathrm{mg} \mathrm{ml}^{-1}$ (Table 1). PCR reactions using the primer pairs ARF4/ARR4 and ARF4/ARS4 amplified a 246-bp product from the QoI-resistant and -sensitive isolates, respectively, verifying the results of the fungicide sensitivity assay (Fig. 1).

Fitness components. All five fitness components showed great variability among isolates within the same sensitivity group (Table 2).

The QoI-sensitive isolates of A. alternata, as a group, had a mean mycelial growth of $40 \mathrm{~mm}$, which was not significantly different $(P$ $=0.21$ ) from the respective mean value for the group of the resistant isolates. Similarly, mean spore production in vitro for the group of the sensitive isolates had a value of $11.8 \times 10^{3}$ spores $/ \mathrm{cm}^{2}$ of medium, which was not significantly different $(P=0.13)$ from the mean spore production in vitro of the resistant isolates which had a value of $15.8 \times 10^{3}$ spores $/ \mathrm{cm}^{2}$ of medium (Table 2).

All of the isolates used in this study, either QoI-sensitive or -resistant, caused disease symptoms on pistachio leaves and produced

Table 1. Sensitivity to azoxystrobin of Alternaria alternata isolates from pistachio used in the study

\begin{tabular}{lcc}
\hline Isolate $^{\mathbf{y}}$ & $\mathbf{E C}_{\mathbf{5 0}}\left(\boldsymbol{\mu} \mathbf{g ~ m}^{\mathbf{- 1}}\right)^{\mathbf{z}}$ & Year of isolation \\
\hline Resistant isolates & & \\
KAC 39 (R1) & $>200$ & 2007 \\
KAC 218 (R2) & $>200$ & 2007 \\
KAC 179 (R3) & $>200$ & 2007 \\
KAC 32 (R4) & $>200$ & 2007 \\
Sensitive isolates & & \\
KAC 2 (S1) & 0.18 & 2007 \\
KAC 42 (S2) & 0.15 & 2007 \\
KAC 27 (S3) & 0.22 & 2007 \\
KAC 22 (S4) & 0.15 & 2007 \\
\hline
\end{tabular}

y Designations R1, R2, R3, R4, S1, S2, S3, and S4 were used in the competition experiments.

${ }^{\mathrm{z}} \mathrm{EC}_{50}=$ effective concentration that reduces spore germination by $50 \%$. spores on the lesions. The first disease symptoms appeared approximately 3 to 8 days after inoculation. However, latent period for the resistant isolates as a group was similar $(P=0.31)$ to that of the sensitive isolates group, with mean values of 4.61 and 5.90 days, respectively. All the isolates, either QoI-sensitive or -resistant, were pathogenic, causing severe disease symptoms on the detached leaves. The Kruskal-Wallis test demonstrated a significant difference in aggressiveness between sensitive and resistant groups at $P=0.011,\left(\chi^{2}=6.35\right)$. Sporulation on the diseased leaf tissues was the last fitness component tested. No significant differences $(P=0.27)$ in sporulation on the diseased tissues were observed between the two sensitivity groups of isolates (Table 2 ).

Competitive ability of QoI-resistant isolates in the absence of fungicide. In the competition experiments, the resistant isolates were better competitors in two of the isolate pairs (R1/S1 and R4/S4), dominating in the population regardless the initial conidial ratios (Fig. 2). In these two pairs, the sensitive isolate disappeared at the third or fourth disease cycle at the $80 \% \mathrm{R}: 20 \% \mathrm{~S}$ ratio. In the remaining two pairs tested (R2/S2 and R3/S3), the sensitive isolates were shown to be better competitors than the resistant isolates (Fig. 2), although the resistant isolate did not disappear in any of the ratios tested.

\section{Discussion}

The recent finding that emergence of fungicide resistance in $A$. alternata is not restricted only to QoIs but has already been expanded to the other important fungicide class of SDHIs necessitates the implementation of anti-resistance strategies aiming to delay selection of QoI-resistant strains. To achieve this purpose, knowledge of the fitness of QoI-resistant strains is a prerequisite.

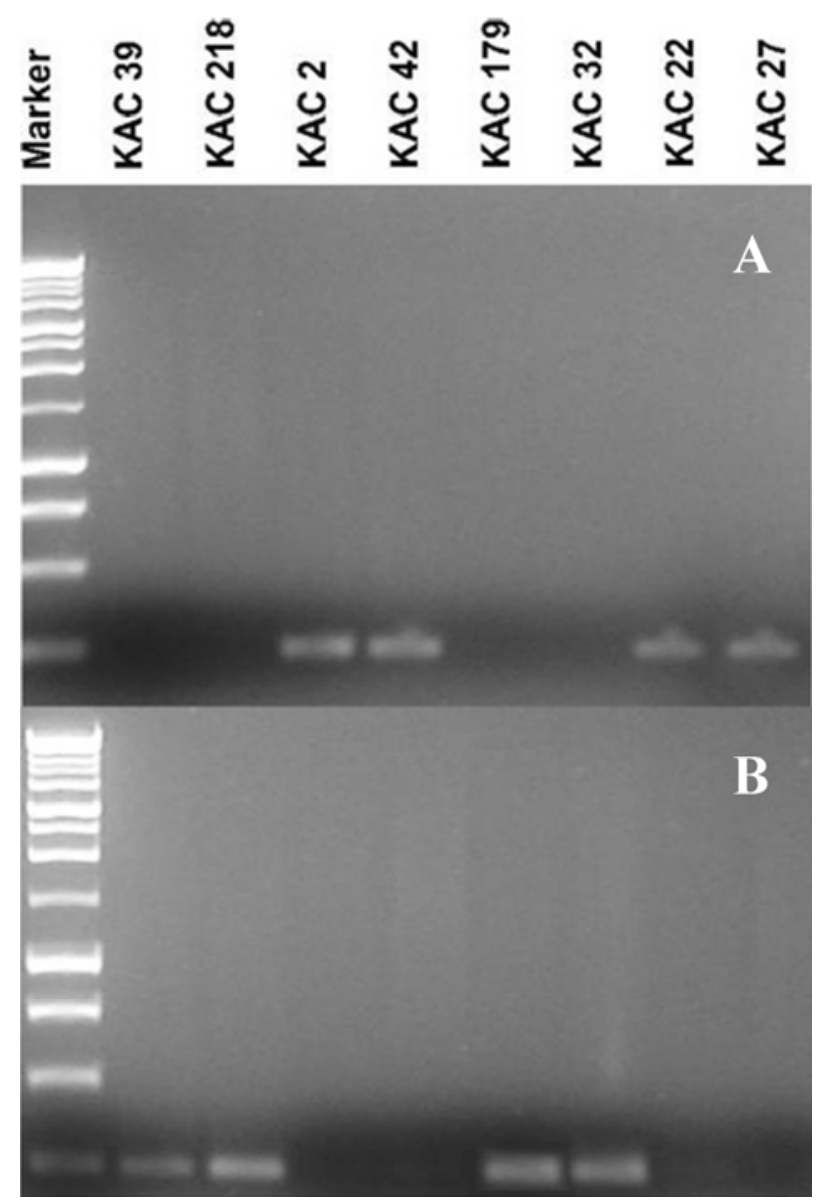

Fig. 1. Detection of azoxystrobin-sensitive (KAC 2, KAC 42, KAC 22, and KAC 27) and azoxystrobin-resistant (KAC 39, KAC 218, KAC179, and KAC 32) isolates of Alternaria alternata from pistachio by allele-specific polymerase chain reaction with the primer pairs A, ARF4/ARS4 and B, ARF4/ARR4, respectively, amplifying a 250bp product. 
In the current study, fitness was evaluated in terms of both "predicted fitness" (measurement of several components in individual isolates) and "realized fitness" (competition between sensitive and resistant isolates in vivo) (1). The competitive ability of the QoIresistant isolates was determined using quantitative allele-specific real-time PCR with the fluorescent dye SYBR Green I. This method is particularly useful to detect different alleles simultaneously and has been used in the past to assess the risk for resistance development to QoIs or to measure the dynamics of QoI resistance in field populations of several fungi $(10,18)$. The quantification feature of the real-time PCR technique represents a powerful tool, enabling its use also for the measurement of resistance-allele frequencies required for the determination of the competitive ability of fungicide-resistant strains. Although, in several studies, competition measurements for QoI-resistant fungal isolates have been performed $(12,17,19)$, the real time-PCR technique has been used in none of them.
Mutations in the cyt $b$ gene associated with resistance to QoIs are accompanied by functionally impaired mitochondria expressing reduced electron flow through the cyt $b c l$ complex, and this may lead to fitness penalties in the strains carrying such mutations $(9,16)$. In fact, several studies related to the fitness and competitive ability of QoI laboratory-induced resistant mutants have shown the existence of a fitness penalty $(24,25)$ but it is more likely that this is due to pleiotropic effects of several accumulated mutations caused during the metallaxigenesis.

When the fitness components of four QoI-resistant and four QoI-sensitive isolates were determined, the resistant isolates appeared to have fitness characteristics similar to those of the sensitive isolates. The results presented in this study showed that, as a group, resistant isolates had latent periods and spore production capacity on host tissues similar to those of the sensitive isolate group, while they showed higher aggressiveness. In addition, resistant isolates, as a group, showed similar mycelial growth and pro-

Table 2. Fitness components of quinone outside inhibitor-resistant and -sensitive field isolates of Alternaria alternata from pistachio

\begin{tabular}{|c|c|c|c|c|c|}
\hline \multirow[b]{3}{*}{ Isolate } & \multicolumn{5}{|c|}{ Fitness components $^{\mathbf{y}}$} \\
\hline & \multicolumn{2}{|c|}{ In vitro experiments } & \multicolumn{3}{|c|}{ In vivo experiments } \\
\hline & Mycelial growth (mm) & Sporulation $\left(\times 10^{3} / \mathrm{mm}^{2}\right)$ & Incubation period (days) & Aggressiveness $^{\mathrm{z}}$ & Sporulation $\left(\times 10^{3} / \mathrm{mm}^{2}\right)$ \\
\hline \multicolumn{6}{|l|}{ Resistant } \\
\hline KAC 39 & $42.58 \mathrm{c}$ & $11.6 \mathrm{~b}$ & $6.87 \mathrm{~b}$ & $2.45 \mathrm{c}$ & $6.3 \mathrm{a}$ \\
\hline KAC 218 & $48.48 \mathrm{~b}$ & $15.7 \mathrm{a}$ & $4.71 \mathrm{~d}$ & $3.10 \mathrm{~b}$ & $1.2 \mathrm{de}$ \\
\hline KAC 179 & $38.08 \mathrm{~d}$ & $9.4 \mathrm{~b}$ & $3.40 \mathrm{e}$ & $3.66 \mathrm{a}$ & $3.5 \mathrm{bcd}$ \\
\hline KAC 32 & $50.50 \mathrm{a}$ & $26.4 \mathrm{a}$ & $3.46 \mathrm{e}$ & $3.65 \mathrm{a}$ & $3.5 \mathrm{bcd}$ \\
\hline Mean & $44.91 \mathrm{~A}$ & $15.8 \mathrm{~A}$ & $4.61 \mathrm{~A}$ & $3.21 \mathrm{~A}$ & $3.6 \mathrm{~A}$ \\
\hline \multicolumn{6}{|l|}{ Sensitive } \\
\hline KAC 2 & $48.00 \mathrm{~b}$ & $5.5 \mathrm{~b}$ & $7.62 \mathrm{a}$ & $2.05 \mathrm{c}$ & $0.57 \mathrm{e}$ \\
\hline KAC 42 & $38.00 \mathrm{~d}$ & $7.2 \mathrm{~b}$ & $5.53 \mathrm{c}$ & $3.71 \mathrm{a}$ & $4.0 \mathrm{abc}$ \\
\hline KAC 27 & $38.75 \mathrm{~d}$ & $23.8 \mathrm{a}$ & $3.95 \mathrm{e}$ & $3.45 \mathrm{ab}$ & $6.1 \mathrm{ab}$ \\
\hline KAC 22 & $35.33 \mathrm{~d}$ & $10.9 \mathrm{~b}$ & $6.53 \mathrm{~b}$ & $2.60 \mathrm{bc}$ & $2.6 \mathrm{cde}$ \\
\hline Mean & $40.02 \mathrm{~A}$ & $11.8 \mathrm{~A}$ & $5.90 \mathrm{~A}$ & $2.95 \mathrm{~B}$ & $3.3 \mathrm{~A}$ \\
\hline
\end{tabular}

${ }^{y}$ Statistical analysis within groups: means of individual isolates followed by the same lowercase letter are not significantly different according to Fisher's protected least significant difference test at $P=0.05$. Statistical analysis between groups: mean values of groups followed by the same uppercase letter are not significantly different according to Student's $t$ test at $P=0.05$.

${ }^{\mathrm{z}}$ Measurements based on a four-point rating system suggested by Pryor and Michailides (30).
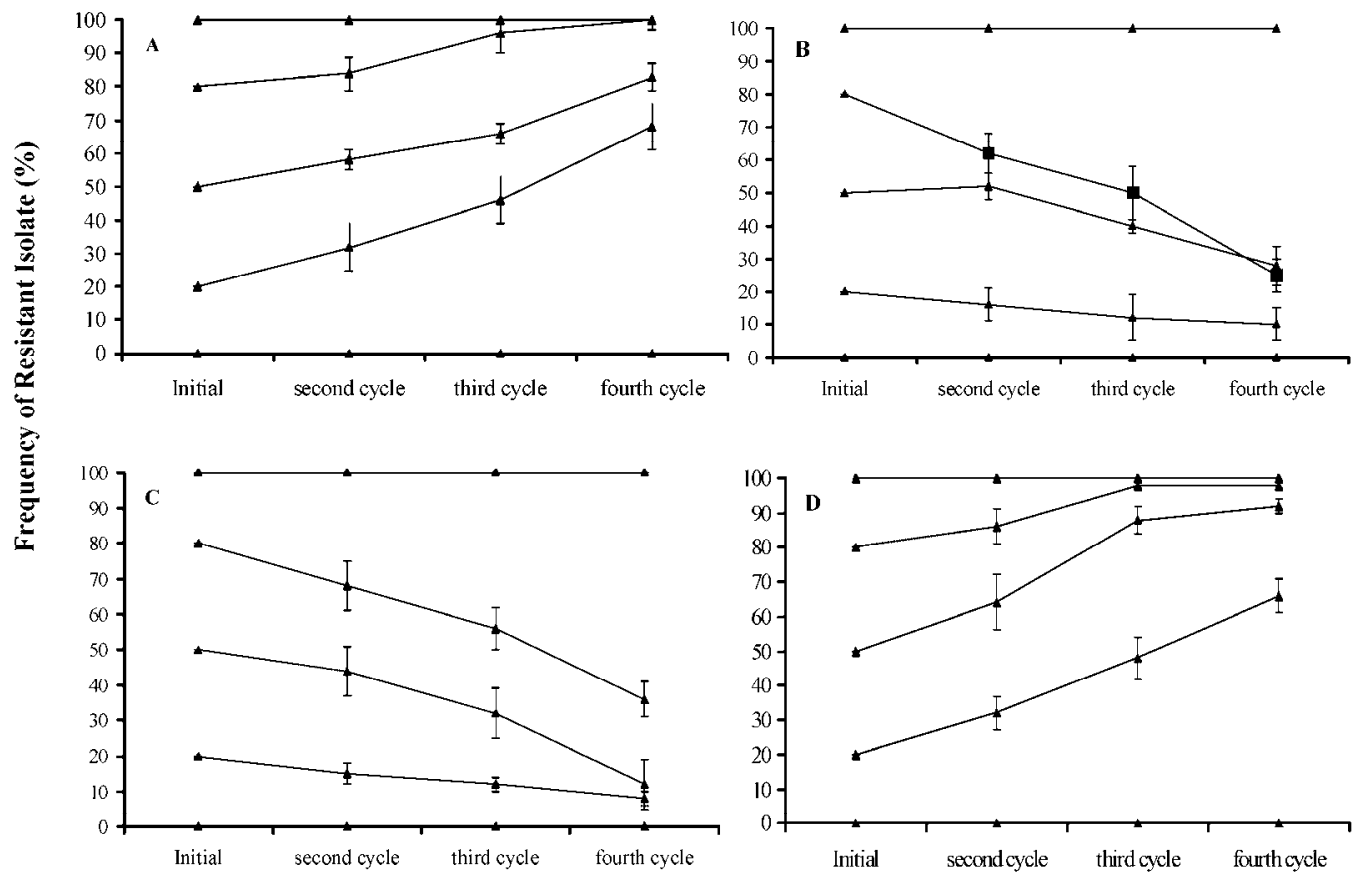

\section{Disease Cycles}

Fig. 2. Competition in vivo between four pairs of azoxystrobin-sensitive and azoxystrobin-resistant isolates of Alternaria alternata from pistachio, co-inoculated on pistachio leaves, with five different initial ratios of conidial mixtures. A, R1/S1 pair; B, R2/S2 pair; C, R3/S3 pair; and D, R4/S4 pair. Vertical lines indicate the standard error of the mean. 
duced similar number of spores on artificial nutrient medium compared with the group of sensitive isolates. However, it should be noted that, within each sensitivity group of isolates, certain variability was observed to several fitness components studied in these experiments. This variability suggests that there is a possibility for selection of highly resistant isolates with high fitness in the field, as has already been reported by Uyenoyama (35). Similarly, the competition experiments between pairs of a sensitive and a resistant isolate showed that the outcome of the competition was isolate dependent. In some pairs, the resistance frequency increased whereas, in others, the resistance frequency decreased, suggesting that the resistant mutants were equally competitive to the sensitive strains. These results confirm the findings of AvilaAdame and Köller (4) and Chin et al. (6), who found an absence of fitness cost in Magnaporthe grisea and Erysiphe graminis f. sp. tritici QoI-resistant isolates, respectively. However, they are in contrast to findings of other reports suggesting decreased fitness and competitive ability in Plasmopara viticola (12) and Magnaporthe oryzae (19) QoI-resistant isolates.

The absence of an obvious fitness penalty in the QoI-resistant strains of A. alternata from pistachio reported in the current study has important implications for disease management. Anti-resistance strategies should aim to slow down resistance frequencies by decreasing the rate of the resistance allele (A143) using fungicides belonging to different chemical classes. Because resistance of the pathogen has already been developed at high frequencies and is not associated with a high fitness cost, the use of QoIs in mixtures or at reduced rates could maintain the selection of the resistance alleles. Although the experiments presented in the current study were carried out under optimal infection and growth conditions, the fitness of the resistant strains under non-optimal infection and growth conditions and the survival ability of the resistant strains during the overwintering period remain unknown. This is particularly important under the light of some recent findings that suggest that the cost of resistance to QoIs may vary with different environmental conditions, being more costly under conditions suboptimal for fungal growth (7). Consequently, further research is required in order to investigate possible fitness costs associated with the Q143A mutation under suboptimal conditions for the growth of A. alternata.

\section{Literature Cited}

1. Antonovics, J., and Alexander, H. M. 1989. The concept of fitness in plantfungal pathogen systems. Pages 185-214 in: Plant Disease Epidemiology, Vol. 2. K. J. Leonard and W. E. Fry, eds. McGraw and Hill, New York.

2. Avenot, H. F., and Michailides, T. J. 2007. Resistance to boscalid fungicide in Alternaria alternata isolates from pistachio in California. Plant Dis. 91:1345-1350

3. Avenot, H., Morgan, D. P., and Michailides, T. J. 2008. Resistance to pyraclostrobin, boscalid and multiple resistance to Pristine (pyraclostrobin + boscalid) fungicide in Alternaria alternata causing Alternaria late blight of pistachios in California. Plant Pathol. 57:135-140.

4. Avila-Adame, C., and Köller, W. 2003. Characterization of spontaneous mutants of Magnaporthe grisea expressing stable resistance to the QoI inhibiting fungicide azoxystrobin. Curr. Genet. 42:332-338.

5. Bartlett, D. W., Clough, J. M., Godwin, J. R., Hall, A. A., Hamer, M., and Parr-Dobrzanski, B. 2002. The strobilurin fungicides. Pest Manage. Sci. 58:649-662.

6. Chin, K. M., Chavaillaz, D., Kaesbohrer, M., Staub, T., and Felsenstein, F. G. 2001. Characterizing resistance risk of Erysiphe graminis f. sp. tritici to strobilurins. Crop Prot. 20:87-96.

7. Cox, K. D., Bryson, P. K., and Schnabel, G. 2007. Instability of propiconazole resistance and fitness in Monilinia fructicola. Phytopathology 97:448453.

8. Fernández-Ortuno, D., Torés, J. A., de Vicente, A., and Pérez-García, A. 2008. Mechanisms of resistance to QoI fungicides in phytopathogenic fungi. Int. Microbiol. 11:1-9.

9. Fisher, N., and Meunier, B. 2008. Molecular basis of resistance to cytochrome $b c_{l}$ inhibitors. FEMS Yeast Res. 8:183-192.

10. Fraaje, B. A., Butters, J. A., Coelho, J. M., Jones D. R., and Hollomon, D. W. 2002. Following the dynamics of strobilurin resistance in Blumeria graminis f. sp. tritici using quantitative allele-specific real-time PCR measurements with the fluorescent dye SYBR Green I. Plant Pathol. 51:45-54.

11. FRAC (Fungicide Resistance Action Committee). 2010. List of resistant plant pathogenic organisms. Available from www.frac.info.

12. Genet, J.-L., Jaworska, T. G., and Deparis, F. 2006. Effect of dose rate and mixtures of fungicides on selection for QoI resistance in populations of Plasmopara viticola. Pest Manage. Sci. 62:188-194.

13. Gisi, U., Sierotzki, H., Cook, A., and McCaffery, A. 2006. Mechanisms influencing the evolution of resistance to QoI inhibitor fungicides. Pest Manage. Sci. 58:859-867.

14. Jeger, M. J., Wijngaarden, P. J., and Hoekstra, R. F. 2008. Adaptation to the cost of resistance in a haploid clonally reproducing organism: the role of mutation, migration and selection. J. Theor. Biol. 252:621-632.

15. Karaoglanidis, G. S., Thanassoulopoulos, C. C., and Ioannidis, P. M. 2001. Fitness of Cercospora beticola field isolates-resistant and -sensitive to demethylation inhibitor fungicides. Eur. J. Plant Pathol. 107:337-347.

16. Köller, W., Avila-Adame, C., Olaya, G., and Zheng, D. 2001. Resistance to strobilurin fungicides. Pages 215-229 in: Agrochemical Resistance-Extent, Mechanism, and Detection. J. M. Clark and I. Yamaguchi, eds. American Chemical Society.

17. Lafarge, D., Abadie, P., Douence, L., Doufour, M., and Corio-Costet, M. 2008. Competitive fitness and adaptation of QoI-resistant Plasmopara viticola strains. Pages 281-285 in: Modern Fungicides and Antifungal Compounds. V. H. W. Dehne, H. B. Deising, U. Gisi, K. H. Kuck, P. E. Russell, and H. Lyr, eds. Deutsche Phytomedizinische Gesellschaft, Braunschweig, Germany.

18. Luo, Y., Ma, Z., Reyes, H. C., Morgan, D. P., and Michailides, T. J. 2007. Using real-time PCR to survey frequency of azoxystrobin-resistant allele G143A in Alternaria populations from almond and pistachio orchards in California. Pestic. Biochem. Physiol. 88:328-336.

19. Ma, B., and Uddin, W. 2009. Fitness and competitive ability of an azoxystrobin-resistant G143A mutant of Magnaporthe oryzae from perennial ryegrass. Plant Dis. 93:1044-1049.

20. Ma, Z., and Michailides, T. J. 2004. An allele-specific PCR assay for detecting azoxystrobin-resistant Alternaria isolates from pistachio in California J. Phytopathol. 152:118-121.

21. Ma, Z., and Michailides, T. J. 2004. A real-time PCR assay for the detection of azoxystrobin-resistant Alternaria populations from pistachio orchards in California. Crop Prot. 23:1259-1263.

22. Ma, Z., and Michailides, T. J. 2004. Characterization of iprodione-resistant Alternaria isolates from pistachio in California. Pestic. Biochem. Physiol. 80:75-84.

23. Ma, Z., Felts, D., and Michailides, T. J. 2003. Resistance to azoxystrobin in Alternaria isolates from pistachio in California. Pestic. Biochem. Physiol. 77:66-74.

24. Malandrakis, A. A., Markoglou, A. N., Nikou, D. C., Vontas, J. G., and Ziogas, B. N. 2006. Biological and molecular characterization of laboratory mutants of Cercospora beticola resistant to Qo inhibitors. Eur. J. Plant Pathol. 116:155-166.

25. Markoglou, A. N., Malandrakis, A. A., Vitoratos, A. G., and Ziogas, B. N. 2006. Characterization of laboratory mutants of Botrytis cinerea resistant to QoI fungicides. Eur. J. Plant Pathol. 115:149-162.

26. Parnell, S., Gilligan, C. A., and Van den Bosch, F. 2005. Small-scale fungicide spray heterogeneity and the coexistence of resistant and sensitive pathogen strains. Phytopathology 95:632-639.

27. Pasche, J. S., and Gudmestad, N. C. 2008. Prevalence, competitive fitness and impact of the F129L mutation in Alternaria solani from the United States. Crop Prot. 27:427-435

28. Peever, T. L., and Milgroom, M. G. 1995. Fungicide resistance: lessons for herbicide resistance management. Weed Technol. 9:840-849.

29. Pringle, A., and Taylor, J. W. 2002. The fitness of filamentous fungi. Trends Microbiol. 10:474-481.

30. Pryor, B. M., and Michailides, T. J. 2002. Morphological, pathogenic, and molecular characterization of Alternaria isolates associated with Alternaria late blight of pistachio. Phytopathology 92:406-416.

31. Raposo, R., Gomez, V., Urrutia, T., and Melgarejo, P. 2000. Fitness of Botrytis cinerea associated with dicarboximide resistance. Phytopathology 90:1246-1249.

32. Sanoamuang, N., and Gaunt, R. E. 1995. Persistence and fitness of carbendazim-resistant and dicarboximide-resistant isolates of Monilinia fructicola (Wint.) Honey in flowers, shoots and fruit of stone fruit. Plant Pathol. 44:448-457.

33. Schoustra, S. E., Slakhorst, M., Debets, A. J. M., and Hoekstra, R. F. 2005. Comparing artificial and natural selection in rate of adaptation to genetic stress in Aspergillus nidulans. J. Evol. Biol. 18:771-778.

34. Sierotzki, H., Frey, R., Wullschleger, J., Palermo, S., Karlin, S., Goodwin, J., and Gisi, U. 2007. Cytochrome $b$ gene sequence and structure of Pyrenophora teres and P. tritici-repentis and implications for QoI resistance. Pest Manage. Sci. 63:225-233.

35. Uyenoyama, M. K. 1986. Pleiotropy and the evolution of genetic systems conferring resistance to pesticides. Pages 207-221 in: Pesticide Resistance: Strategies and Tactics for Management. National Research Council, Committee on Strategies for the Management of Pesticide Resistant Pest Populations, eds. National Academy Press, Washington, DC. 